\title{
Using Network Analysis to Discover Cooperation Opportunities in Inter-organizational Networks
}

\author{
Laura Ponisio ${ }^{1}$, Pascal van Eck ${ }^{2}$, Lourens Riemens ${ }^{3}$, and Noriyuki Matsuda ${ }^{4}$ \\ 1 BE Software Design, Amsterdam, The Netherlands \\ ml@ponisio.com \\ ${ }^{2}$ University of Twente, Enschede, The Netherlands \\ pascal@pascalvaneck.com \\ 3 Dutch Tax and Customs Administration, Apeldoorn, The Netherlands \\ lj.riemens@belastingdienst.nl \\ ${ }^{4}$ University of Tsukuba, Ibaraki, Japan \\ mazda@sk.tsukuba.ac.jp
}

\begin{abstract}
In a network of organizations, members are often faced with the problem of choosing partners for closer cooperation within this network. Consequently, network members collect information about potential partners to reach informed decisions about for instance starting new joint development projects or harvesting best practices. The large amounts of information involved in these decision processes obscure possibilities, and choices are made ad hoc. In this paper, we present an approach that uses techniques from network analysis to support organizations in processing and understanding this information. Central in our approach are network visualizations that help in comparing gaps between the aspired and current development levels of the processes of the member organizations. The advantage of our approach, which we validated via expert interviews, is that such visualizations are generated semi-automatically and offer an overall view of the current and aspired situation in the network without losing the ability to pinpoint particular, individual processes of interest.
\end{abstract}

\section{Introduction}

In today's networked world, organizations seek to improve their IT processes and systems by collaborating with partners in any network they participate in. For example, two organizations may jointly develop a shared online transaction processing system with the objective of reducing costs and harmonizing procedures. However, finding a partner for closer cooperation has several challenges [15]. Firstly, investigating possibilities for closer cooperation in all but the smallest networks results in too much information for decision makers to process in detail; which leads to arbitrary decisions. Secondly, this decision process is very costly and brings long-term consequences.

The purpose of this paper is to explore the potential of network analysis [6, 2] to find partners for closer cooperation with the final objective of improving IT processes and systems of the organizations involved. To this end, this study answers the following question: Is network analysis a useful instrument to find partners with whom to cooperate closer in an inter-organizational network? To answer this question, we put network 
analysis to test in the domain of eCustoms: a network of 27 European Union (EU) member states that have the same legislation, and have the objective to act as one monolithic customs organization in their relation with traders. Using an action research method in a case at the Dutch Tax and Customs Administration, we devised an approach based on network analysis theory to support choosing partners based on improving understanding of the current development level and improvement aspirations of the organizations.

Our results indicate that our approach has the potential to help eCustoms decision makers to find prospective alliances between customs organizations of EU countries. In fact, our network analysis method has, in the domain of eCustoms, the potential to build a workable solution to the problem of finding a partner. In this study, we show how network analysis techniques made it possible to build automatic visualizations that offer an overall view of the current and aspired situation in the network without loosing the ability to pinpoint particular, individual processes that would not be visible in traditional analysis methods. Having our results confirmed by expert interviews, we conclude that network analysis could be a useful instrument for revealing partners for closer cooperation. Moreover, our results indicate that software process improvement (SPI) may benefit from an improved understanding of organizations in an inter-organizational, alliance formation context.

\section{SPI in an Inter-organizational Context}

The fundamental objective of SPI is to change software development processes in order to achieve improvements in quality and productivity [1]. Several works have attempted to make an integrated body of literature [7, 9], while other research efforts focused on studying factors that influence success of SPI programs [11, 8]. Results assert the importance of adapting metrics programs to their context: to be successful, the programs implemented should be defined according to the focal organization's specific information needs [14]. In fact, research has clearly shown that success in SPI efforts depends on focusing on the needs of the organization in question [1, 13].

In practice we observe a newly emerging context for software process improvement, where organizations seek to jointly develop capabilities in symmetric relations, without a clear distinction between vendor and customer, and at least initially without the clarity provided by a formal contract. IT organizations form (strategic) alliances. Which mechanisms will organizations use to improve systems in the context of such alliances?

\subsection{Alliance Formation}

In finding partner for cooperation, organizations can follow two strategies: exploitation and exploration [16]. According to alliance theory [12], organizations need to be able to use both strategies (which is called 'ambidexterity'). Researchers have developed several measurement instruments to determine the strategic orientation of an organization. For instance, He and Wong [10] developed four Likert-scale items that characterize exploitation and four Likert-scale items that characterize exploration, related to objectives of innovation efforts while Popadiuk [20] uses a different technique to determine the strategic orientation of an organization. His measurement instrument illustrates the breadth of the ambidexterity concept. 
Whereas such instruments are useful for empirical research, the increase in complexity of alliances calls for scalable methods that can deal with the many factors involved. In the area of inter-organizational (IO) networks, researchers have recognized the need for an integrative model of cooperation in an IO context and works that supports practice [5]. A survey of network analysis from the management perspective found that the research field is populated with insights derived from micro analysis, but that it was lacking a macro level that would explain processes in the whole network [12].

A case in point is eCustoms, a large distributed system that connects the customs organizations of a number of member states of the EU [19, 18]. Which strategy should member states follow to find partners for closer cooperation? According to He and Wong's objectives, eCustoms could follow an exploitation strategy to find partner for cooperation, for eCustoms seeks to reduce development costs and to learn from each other's best practices. But which concrete, practical techniques can eCustoms members use to determine the inter-organizational ability of potential partners for closer cooperation? To the best of our understanding, such techniques have not yet been explored.

\section{Research Method}

The methodological approach we used in this paper follows the guidelines of Baskerville [3] on methods for carrying out action research. We have chosen action research because we wanted to investigate whether a solution we propose proves to be useful when applied in an actual case in practice. This case is the eCustoms benchmark (see Section 3.1). Experts at the Dutch Customs Administration informed us about the challenges they were facing in finding partners for closer cooperation and shared with us the data of a benchmark performed by them.

The first step in our action research approach comprised problem investigation, which suggested the need for a method to give decision makers more insight about the eCustoms situation in terms of the current and aspired state of processes and supporting IT. As this can be very complex, the method should be semiautomatic and efficient, supporting the decision-making process with visualization and measurements. Consequently, the goal of our research was to define an approach with which a global view of eCustoms data can be built semi-automatically and to evaluate this approach in practice. Because network analysis has proven useful to find the underlying structure behind complex networks in the most diverse research areas [17, 4], we hypothesized that network analysis can be used as the basis for our approach.

The next step in our research was to design a network-analysis based approach with which visualizations of the eCustoms data can be constructed semi-automatically. The approach takes the form of a pipeline of data processing tools. The first stage of this pipeline consists of a program created by us that reads the benchmark data and generates a graph in the GML format, based on the representation introduced in Section 4. The second stage loads that graph in networkX (networkx.github.com) and runs some centrality measurements, gathering insight on each customs organization.

The final stage is displaying the visualization, for which we use Gephi (http://gephi.org) and YinfanHu's algorithm. We applied our approach to the eCustoms benchmark data to find potential partners for closer cooperation (Section 5) 
and discussed our insights in expert interviews to see if, in the perception of experts, the global view provided by our visualization is indeed easier to grasp than a long list of data provided by the conventional analysis techniques used in the benchmark project (Section 6).

\subsection{The eCustoms Case}

To study how our approach could be useful to find partners for closer cooperation in an existing inter-organizational network, we applied our method to a concrete instance of such a network: eCustoms. In this network of customs organizations, small groups of members try to improve their supporting IT processes and systems by closely cooperating with one another.

This network was established by the European Commission, aiming to improve ensuring safety of the external borders of the EU, and to facilitate trade. All customs organizations of the 27 member states of the EU belong to this network. eCustoms has been in place for many years, but new demands make it necessary to cooperate closely. Recognizing information is key to find opportunities for collaboration, the Dutch Tax and Customs Administration conducted between January and October 2005 a study that compared business processes, systems and ambitions of ten member states. The benchmarking study provided the data needed to analyze opportunities for cooperation.

\subsection{The eCustoms Benchmark Data}

In our research, we focused on the business process development aspect of the data, consisting of the current and aspired development levels of 53 processes distributed over areas such as declaration handling, inspections, post arrival processing, account management, risk management, collaboration and partnerships and enterprise services. Of these processes, we focused on the 42 that had quantitative answers (processes do not have consecutive numbers in our descriptions and figures because we left out the 11 qualitative items). For each of these processes, a local expert of the customs organization had completed the survey by supplying an assessment of a current and aspired level. The scale of levels was from one to five.

For example, The Netherlands (NL) assigned a five to the current level of process 28, Calculate duties, and a five to its aspired level. As both levels are equal to each other and to the highest level possible, NL thus indicated it sees no need to improve this process. Process 53, Share information within the organization, tells a different story: it has current level two and aspired level four, indicating interest of NL to improve it. This circumstance opens the door for decision makers to search collaboration with other customs organizations to join forces in improvement projects.

Traditional statistical analysis of the 42 processes with quantitative data revealed that not all processes have the same importance for the Dutch Customs Administration. We performed this traditional analysis in a phase in which we investigated the eCustoms case from many different angles (a phenomenon common to action research) before converging on network analysis. The areas NL are more interested in to develop are Collaboration and partnerships and Shared information within the organization. This insight is used in Section 5.2 . 


\section{Network Approach for Analysis and Visualization}

We constructed a directed network for each country using the 42 quantitative items of the survey as the process nodes $P_{i},(i=1, \ldots, 42)$. They are linked to the level nodes $L_{j},(j=1, \ldots, 5)$, according to the current and aspired ratings of the items. More specifically, edges from $L_{j}$ to $P_{i}$ and from $P_{i}$ to $L_{k}$ represent the current and aspired relationships, respectively.

In theory, $P_{i}$ is connected from $L_{j}$ and to $L_{k}$ in one of three ways: 1) $j=k$, which means that $P_{i}$ is bidirectionally connected to $L_{j}=L_{k}$ (in this case we say $P_{i}$ is a static node), 2) $j<k$, which means that the current level of $P_{i}$ is lower than its aspired level (we say $P_{i}$ is an upgrading node), or 3) $j>k$, which means that the aspired level of $P_{i}$ is lower than its current level (we say $P_{i}$ is a downgrading node). This third possibility does not appear in our case study and we think it is very rare in practice.

Example. Figure 1 illustrates the application of our method to visualize 42 customs processes of NL. Each process is linked to its current and aspired levels, which are represented by five level nodes, making 47 nodes altogether. To facilitate reading, in these graphs a node is bigger if it is a level node. In our eCustoms examples, the labels of the process nodes range from 1 to 53 (rather than from 1 to 42) in correspondence with the numbers of the questionnaire items. As 11 qualitative items do not appear in the graphs, the numbers are not consecutive.

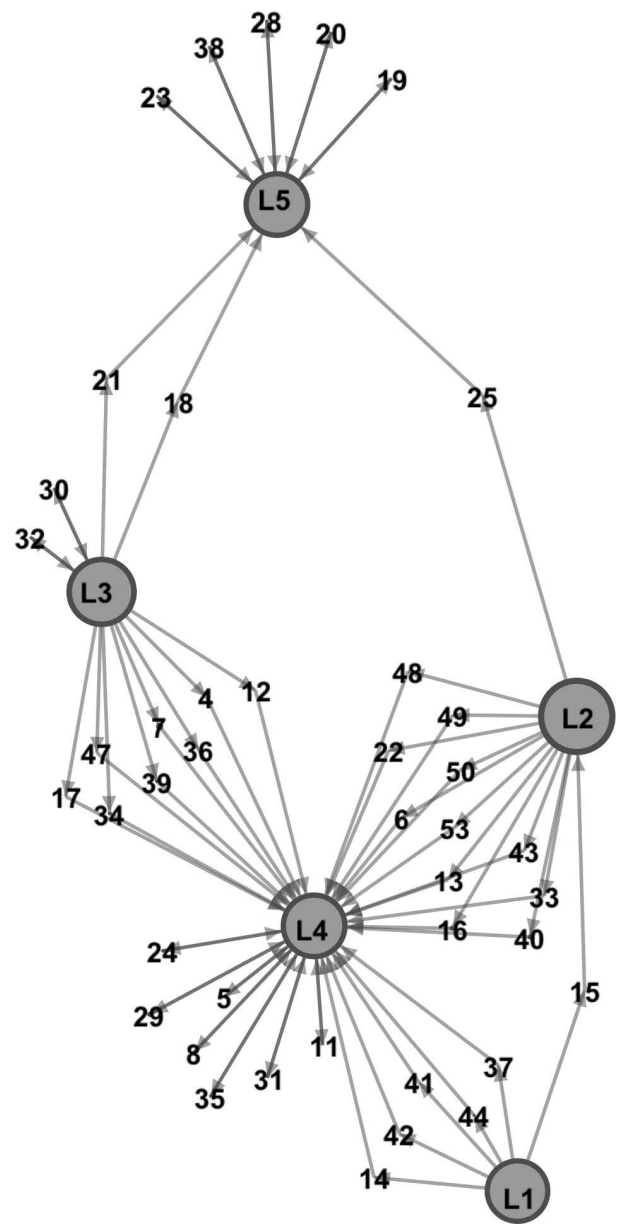

Fig. 1. Network representation of the survey results for $\mathrm{NL}$

Static Nodes. Processes that are static nodes have reached their maximum level according to what the country finds interesting. At the top of the graph in Figure 1, we see a group around the node with label L5 (processes 19, 20, 23, 28 and, 38). These nodes indicate processes that are already developed (their current level is the same as the aspired level), and there is no need to search for a partner that can teach how to improve on these processes in a technology-transfer relationship. 
Further verification revealed that almost all static processes in NL were processes that support the top three IT issues the Dutch Customs Administration was facing at the time of the benchmarking: 1) improve IT processing efficiency and productivity, 2) standardize IT environment, and 3) online government and e-services. For instance, process 28, Calculate duties, is essential for customs activities.

Process 30 (Forecast revenue impact) and 32 (Link manifests with declarations) are static nodes and at a lower level (three). The fact that they are static nodes indicates little interest of improvement, which is in line with the fact that these processes only slightly affect either transaction performance, or the top three IT issues the Dutch Customs Administration is facing.

In Figure 1, NL shows advanced essential customs processes. This is in line with what we observed of their customs performance, as NL has one of the biggest entry points in the EU (the Rotterdam harbor). The benchmark study showed that at the time of the benchmark, NL processed the second highest percentage of transit declarations per year in the whole sample, and therefore has high availability requirements. Without advanced essential customs processes, NL would be unable to have the required capacity and robustness.

In terms of opportunities for closer cooperation, the static-nodes-at-a-high-level pattern tells us a good partner for NL would have a high current level for these processes, as well.

Upgrading Nodes. Processes that are upgrading nodes have their current development level lower than their aspired level. Improvement is desired there. In Figure 1, these nodes are connected to a current level node that is lower than their aspired level node. Most processes are upgrading nodes: their current level is either L1, L2 or L3, and many have aspired level L4, which tells us most of the processes are desired to improve. A case in point is process 53, Share information within the organization. Located between L2 and L4, upgrading node 53 indicates NL is interested in improving this process.

The graph shown in Figure 1 suggests that experts need to focus on the processes linked to level 4 as their aspired level. This result suggests also to look for a symmetric partnership.

Process 25, Administer the exit of goods, in Figure 1 is exceptional: it is the only process NL wants to improve from 2 to 5 (the highest level). Its location in the graph draws the attention of decision makers to its uniqueness. This can be made formal using the network analysis concept of betweenness centrality. The graph shows also two other exceptions: processes 18, Administer the entry of goods, and 21, Administer the transit of goods. Together with process 25 , these processes have in common that they belong to an essential area of NL and their aspired level is way higher than their current level. They all belong to the area Declaration Handling, an important area in customs that becomes crucial to improve when NL wants to be paper free and more efficient.

\section{Visualization Findings: Potential Partners for Closer Cooperation}

In this section, we show how our network approach for analysis and visualization supports the task of finding a partner for closer cooperation in several ways. Firstly, we 
show how potential partners or non-partners can be identified by inspecting the shape of their respective graphs (Section 5.1). We then zoom in on closer inspection of the graphs of countries identified as potential partners of NL (Section 5.2).

\subsection{Comparing Countries}

Two countries have good perspectives to succeed in an effort for closer cooperation if the current level and ambitions of their processes match. In order to match, two partners must have important processes (whichever those are for each country) developed to a similar extent. For new development, it is desirable that processes are at a similar level. To harvest best practices, it is desirable to look for a partner with a higher current level. According to eCustoms experts, "The bigger the overlap [in processes], the faster we can work.", and, "If processes don't fit, closer cooperation is going to be very difficult." Both partners must be interested in improving approximately the same set of processes, since closer cooperation requires similar interests to justify the investments needed. In terms of our visualizations, this means that we look for similar patterns around single processes, e.g., the occurrence of upgrading nodes in two graphs for the same process.

Thus, two countries have poor perspectives to succeed in an effort for closer cooperation if the current level and ambitions of their processes do not match. A case in point is shown in Figure 2, where Country A is very different from Country B. For instance, Country B has no process at level 1 (L1 is isolated at the bottom left) and Country B's most processes and systems are level 5 or level 4 . In contrast, Country A has no such concentration of processes at high levels.

Conversely, Figure 2d and Figure 2c show an example of two countries with good prospects of closer cooperation: Country $\mathrm{C}$ and Country D have the same structure in term of current and aspired level of their customs processes.

\subsection{Partners under the Magnifier}

When considering a network member as a partner for closer cooperation, it is particularly important to know how the potential partner performs in the processes that are essential to our own organization. A key question is: How advanced are our essential processes on their side? We used this reasoning to better understand which network members are better partners for NL.

Firstly, we need to know which processes are important for our organization. We found the most important processes for NL (Section 3.2) and highlighted them in the network representation of this country. Figure $3 \mathrm{a}$ shows a visualization of NL where the aspiration links for important processes are highlighted.

Secondly, we need to know how potential partners perform. To achieve this goal, we built the network representations of other customs organizations in the same way we have seen in Section 4 and highlighted in those graphs the processes that are important for NL. In the graph, an edge is bigger if it belongs to an area that NL is interested to develop. Figure 3 shows the resulting visualization of Country A and Country E.

Country A. Figure $3 \mathrm{a}$ and Figure $3 \mathrm{~b}$ show that NL and Country A are interested on developing different things. Drawing Country A from the perspective of NL's needs, 


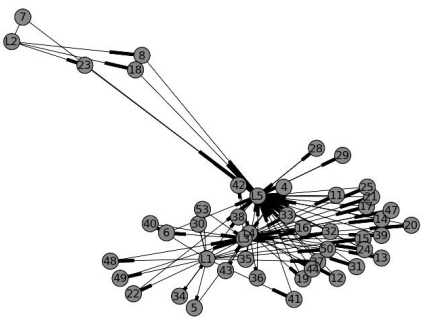

(a) Country A

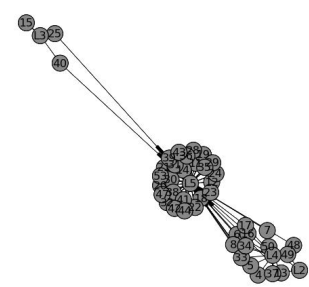

(b) Country B Two countries with poor prospects of closer cooperation

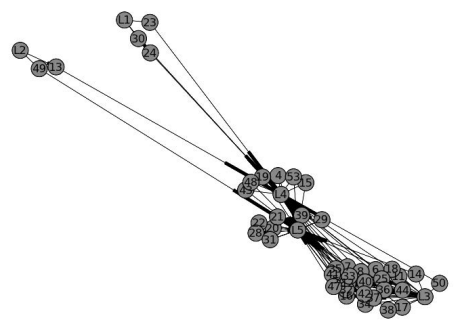

(c) Country C

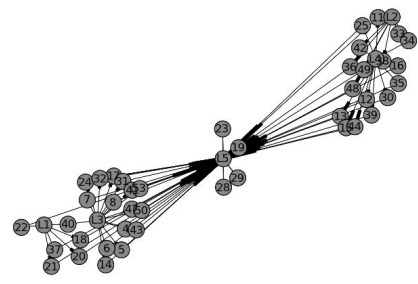

(d) Country D

Two countries with high prospects of closer cooperation

Fig. 2. Network interpretation of survey results for several countries

Figure $3 \mathrm{~b}$ reveals that the processes that are important to NL (thick arrows) are distributed all over the graph of Country A.

In particular, NL is interested in improving 11 processes, only four of which are at a high level in Country A (i.e., 39, 41, 43 and 44). Moreover, five processes important for NL are currently at the lowest possible level of development in Country A, level 1 (i.e., 40, 48, 49, 50 and 53). Since some areas of interest to NL appear in the Country A network representation as static nodes (47 and 43), we learn Country A does not aspire to improve the levels of these areas and therefore it is probably not interested in forming a closer partnership to improve them.

In short, Figure 3 tells decision makers that Country A and NL are too different to make promising close partnership to improve processes in a symmetric bi-directional relationship (at best, they could build an asymmetric technology-transfer partnership).

Country E. Figure 3c depicts a network representation of Country E, a better partner for NL. As in Figure 3a and Figure 3b, thick arrows represent the processes that are important to NL. Most of the processes highlighted are pointing to level 5, meaning that Country $\mathrm{E}$ is interested in improving almost every single process NL finds important and that the current level of these specific processes are already at a high level (4). 


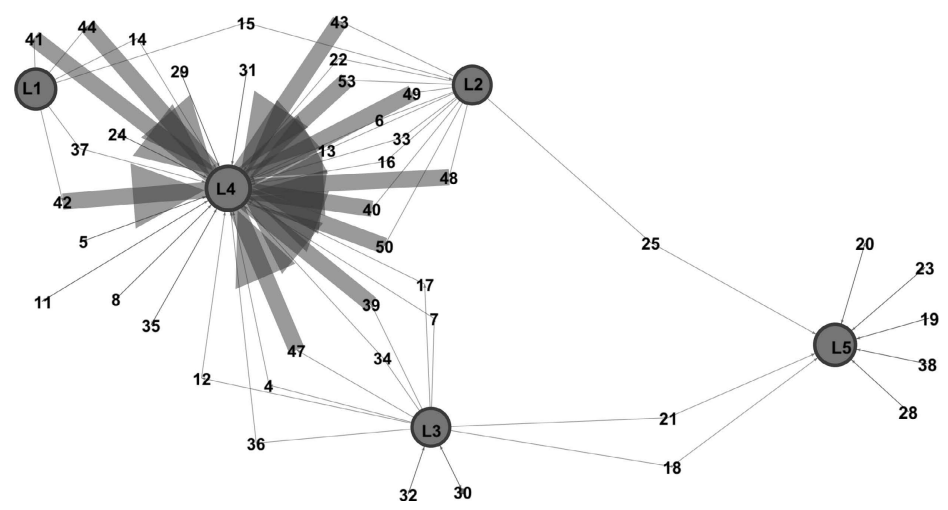

(a) NL

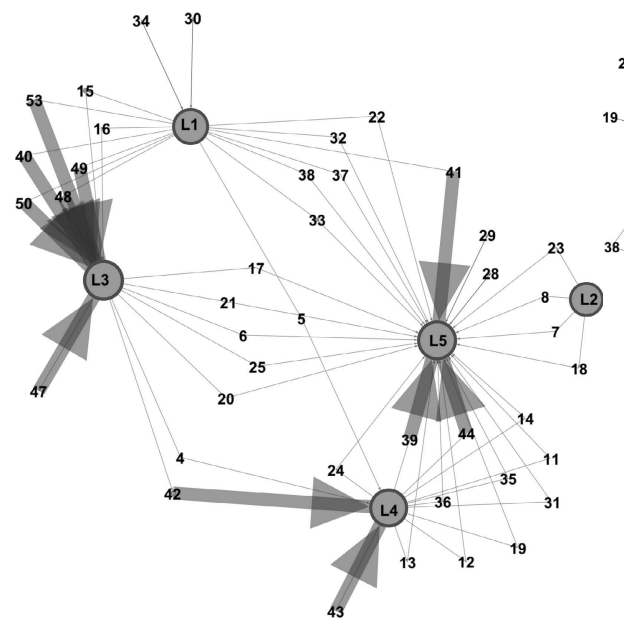

(b) Country A

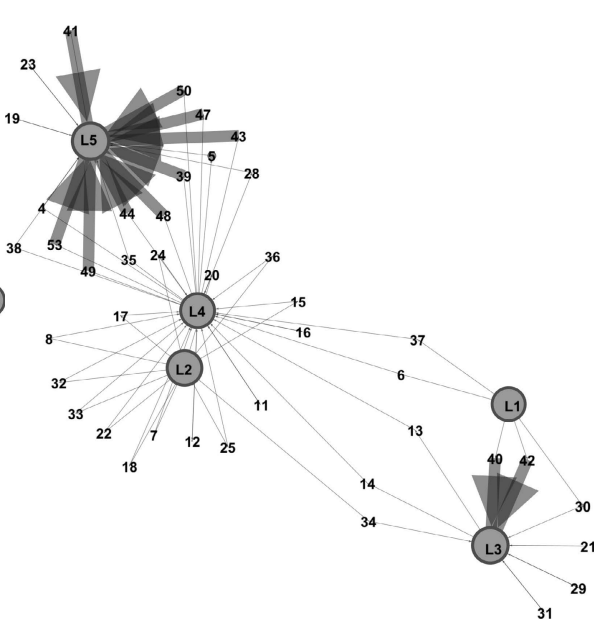

(c) Country E

Fig. 3. The current and aspired values of the essential processes in NL, Country A and Country E

Process 41 is a static node connected to L5, which means Country E already has process 41 at a high level. Thus NL (where process 41 has current level L1 and aspired level L4, as shown in Figure 3a), could profit from the experience of Country E.

Processes 40 and 42 (upgrading nodes linked to L3 in Figure 3c) show that NL and Country E could benefit from a partnership because although at a slightly higher level in $\mathrm{NL}$, both countries aspire to upgrade each of these processes (the corresponding nodes in Figure 3 a are upgrading nodes connected to L4).

Altogether, the situation of processes 40, 42, and 41 suggests that a symmetric bidirectional technology-transfer partnership is a possibility, where NL can benefit for process 41 and Country E can benefit for processes 40 and 42 . Together with the other processes, Figure 3 shows that NL and Country E have matching current levels and aspirations for essential processes, which might be evidence of a plausible successful closer partnership between NL and Country E. 


\section{Case Study Results: Expert Perception of Visualization Insights}

Applying our approach produced two visualizations. Firstly, we saw in Figure 2 a network interpretation for four countries: two countries with poor potential for closer cooperation (Figure $2 \mathrm{a}$ and Figure 2b), and two countries with good potential for closer cooperation (Figure 2c and Figure 2d). Secondly, we saw in Figure 3 that our representation indicates that the customs organizations of NL and Country A are very different, hindering possibilities of successful cooperation and that the customs of NL and Country E have interests that fit with one another.

These results have been discussed with customs experts who had participated in the original benchmarking study. The experts found the reasoning leading to the identification of potential cooperation partners surprisingly correct, and proposing Country E as a good potential partner for NL matched their insight.

\subsection{Implications for Practice}

According to Dutch experts, our approach is useful to find potential candidates for closer cooperation because, firstly, it supports decision makers in dealing with the large amount of information. The advantage of this method is that it helps decision makers to process a lot of data. "There are too many potential partners and too much information. With three partners you can keep it all in your head, but for more partners you need something like this."

Moreover, manipulation of the graph, for instance highlighting all the processes related to an area, helps decision makers to discuss the common points between two partners. They noted that depending on the area of collaboration, a partial match might be good enough.

Secondly, our method is helpful to find potential partners for closer cooperation because it is explicit, pointing out the rationale behind the choice of a potential partner. "A lot we do is intention based. We think it would be good to talk with [country X]. There is much politics. It would be good to base collaboration more on attributes such as the architecture possibilities."

Thirdly, experts noted that our network approach for analysis to find partners for closer cooperation has good potential for scalability, since our method could be extended with new attributes such as language and geographical location of the country. Semi-automatization makes it possible to scale up, since our approach makes it easy to include more countries and get visualizations instantly. "[The ability to effortlessly include new countries easily is required because] we have now new countries that want to participate."

\subsection{Discussion of Our Approach}

Novelty. Like other approaches, our approach supports decision makers in discussing opportunities for cooperation. However, our model goes beyond the theoretical plane by exploiting tools to analyze a great amount of information without loosing neither the big picture, nor the zoom-in lens. This sets our approach apart from traditional methods. 
Generalization to other networks. Data center consolidation (different issue, different network) and IT services in a network of institutions of higher education (same issue, different network) are examples of areas mentioned in expert interviews where our approach would be useful. Thus, we cannot prove that our research generalizes to other cases, but the results of our case study are encouraging at the very least. Experts consulted were positive about this, considering our method useful to detect opportunities for cooperation in networks where partners could define their ambitions.

Future work. While the graphs presented in this paper are generated semi-automatically, currently in our approach the identification of suitable partners for closer cooperation includes manually assessing the (dis)similarity of these graphs. Our approach could be enhanced by applying similarity metrics to discard some graphs, such that only promising candidates are left for manual inspection.

A second line of future work is about different scenarios for collaboration. Collaborating closely is much more than copying. When planning closer cooperation, what should we take from a partner? Should we copy their source code, their design or a functional idea of data-process separation? Our current network graphs such as those presented in this paper are not about source code or systems. However, there is enough evidence to believe our method can potentially be helpful here, too. We expect that we will encounter additional dimensions apart from the two we currently address (current and aspired level and the gap between these) and that we will have to extend our visualizations accordingly, e.g. by using size and/or color of nodes and edges.

\section{Conclusion}

We conclude that a network-analysis based approach for decision support in alliance formation within existing networks is a useful tool for determining potential partners for closer cooperation in such a network. The visualizations that our semi-automatic network-analysis based approach enables reveal similarities and dissimilarities that are the basis for selecting potential partners. This is the starting point for further strategic planning by each of the potential partners. Our approach proved to be able to process large amounts of information.

Acknowledgment. We thank the anonymous reviewers for their valuable comments.

\section{References}

[1] Aaen, I., Arent, J., Mathiassen, L., Ngwenyama, O.: A conceptual MAP of software process improvement. Scandinavian J. of Inf. Sys. 13, 81-101 (2001)

[2] Barabasi, A.L.: Linked. Perseus Publishing (2002)

[3] Baskerville, R.: Investigating information systems with action research. Communications of the Association for Information Systems 2, 4 (1999)

[4] Borgatti, S.: Identifying sets of key players in a social network. Comp. \& Math. Org. Theory $12,21-34$ (2006)

[5] Chi, L., Holsapple, C.: Understanding computer-mediated interorganizational collaboration: a model and framework. J. of Knowl. Management 9, 53-75 (2005) 
[6] Freeman, L.: Centrality in social networks conceptual clarification. Social Networks 1(3), 215-239 (1978)

[7] Fuggetta, A.: Software process: a roadmap. In: ICSE 2000: Proc. Conf. on the Future of Software Engineering, pp. 25-34. ACM, New York (2000)

[8] Goldenson, D.R., Herbsleb, J.D.: After the appraisal: a systematic survey of process improvement, its benefits, and factors that influence success. Technical CMU/SEI-95-TR-009, Softw. Eng. Institute, Carnegie Mellon Univ. (1995)

[9] Hansen, B., Rose, J., Tjørnehøj, G.: Prescription, description, reflection: the shape of the software process improvement field. Int. J. Inf. Mngt. 24(6), 457-472 (2004)

[10] He, Z.L., Wong, P.K.: Exploration vs. exploitation: An empirical test of the ambidexterity hypothesis. Organization Science 15(4), 481-494 (2004)

[11] Herbsleb, J., Zubrow, D., Siegel, J., Rozum, J.: Software process improvement: State of the payoff. American Programmer 7, 2-12 (1994)

[12] Hoffmann, W.H.: Strategies for managing a portfolio of alliances. Strategic Management Journal 28(8), 827-856 (2007)

[13] Iversen, J., Ngwenyama, O.: Problems in measuring effectiveness in software process improvement: A longitudinal study of organizational change at danske data. Int. J. Inf. Mngt. 26(1), 30-43 (2006)

[14] Kautz, K.: Making sense of measurement for small organizations. IEEE Softw. 16(2), 14-20 (1999)

[15] Kumar, K., van Dissel, H.G.: Sustainable collaboration: Managing conflict and cooperation in interorganizational systems. MIS Quarterly 20(3), 279-300 (1996)

[16] Lin, Z.J., Yang, H., Demirkan, I.: The performance consequences of ambidexterity in strategic alliance formations: Empirical investigation and computational theorizing. Management Science 53(10), 1645-1658 (2007)

[17] Matsuda, N., Takeuchi, H.: Networks emerging from shifts of interest in eye-tracking records. eMinds: Int. J. Human-Computer Interaction 2(7), 3-16 (2011)

[18] Ponisio, L., van Eck, P., Riemens, L.: Planning cooperation in inter-organizational systems. In: E-Strategies for Resource Management Systems, pp. 61-85. IGI Global (2010)

[19] Ponisio, M.L., Sikkel, K., Riemens, L., van Eck, P.: Combining visualisation techniques to understand co-operation in inter-organisational systems. Journal of Systems and Information Technology 10(2), 159-179 (2008)

[20] Popadiuk, S.: Scale for classifying organizations as explorers, exploiters or ambidextrous. International Journal of Information Management 32(1), 75-87 (2012) 